\title{
Quantification of thymol content in different extracts of Zataria multiflora by HPLC method
}

\author{
Azar Aghamohammadi ${ }^{1}$, Mohammad Azadbakht ${ }^{1,2}$, Seyed Jalal Hosseinimehr ${ }^{1,3^{*}}$ \\ ${ }^{1}$ Traditional and Complementary Medicine Research Center, Mazandaran University of Medical Sciences, Sari, Iran \\ ${ }^{2}$ Department of Pharmacognosy, Faculty of Pharmacy, Mazandaran University of Medical Sciences, Sari, Iran \\ ${ }^{3}$ Department of Radiopharmacy, Faculty of Pharmacy, Mazandaran University of Medical Sciences, Sari, Iran
}

Received: Feb 2, 2016, Revised: Feb 20, 2016, Accepted: March 6, 2016

\begin{abstract}
Zataria multiflora Boiss is used in traditional folk remedies as antiseptic, analgesic, carminative, anthelmintic medication. The main components of $Z$. multiflora are phenolic compounds such as thymol and carvacrol. The aim of this study was developed a simple and rapid method for determination of the thymol in different extracts of Z. multiflora by HPLC method. The dried aerial parts of Z. multiflora in the flowering stage was extracted with ethanol $34 \%, 42 \%$ and $70 \%$ for 48 hours. Several mobile phase systems were applied for development and separation of thymol and carvacrol that have close retention time in HPLC system. The peaks of thymol and carvacrol are successfully separated in acetonitrile-water-acetic acid mobile phase. Thymol and carvacrol were separated with a retention times 10.4 and 9.8 minutes respectively, in an isocratic solvent system with HPLC. Thymol content was $2.7 \pm 0.06$, $3.7 \pm 0.07$ and $6.0 \pm 0.11 \mathrm{mg} / \mathrm{g}$, in ethanol at concentration of $34 \%, 42 \%$ and $70 \%$, respectively. Thymol content in different hydroalcoholic extract of $Z$. multiflora is dependent to ethanol concentration in extraction solvent.
\end{abstract}

Keywords: Z. multiflora, thymol, carvacrol, HPLC

Pharm Biomed Res 2016; 2(1): 8-13

DOI: 10.18869/acadpub.pbr.2.1.8

\section{Introduction}

Lamianceae family is one of the largest herbals' families that are growing in the world and has 200 genus and 2000-5000 species of aroma. Zataria multiflora Boiss. (ZM) (synonyms: Zataria bracteata Boiss.; Zataria multiflora var. elatior Boiss) is a thyme-like plant and a member of this family with multiple, thin, hard and forked stalks (1,2). ZM is containing thymol, carvacrol, zatrinal, oleanolic acid, betulic acid, rosmarinic acid and monoterpenoids such as sesquiter-penoids, p-cymene and terpinene (2). The main components of $Z M$ oil are phenolic compounds. The biological effects of $Z$. multiflora are mainly associated to its phenolic compounds, especially thymol and carvacrol (Fig. 1) (3). ZM has several biological and pharmacological properties including anti-fungal (4), antierythma (5), anti-nociceptive (6), anti oxidative stress $(7,8)$, antimicrobial $(2,9)$ spasmolytic, anti-inflammatory (10), immunostimulant (11) painrelieving (12) and radioprotective 


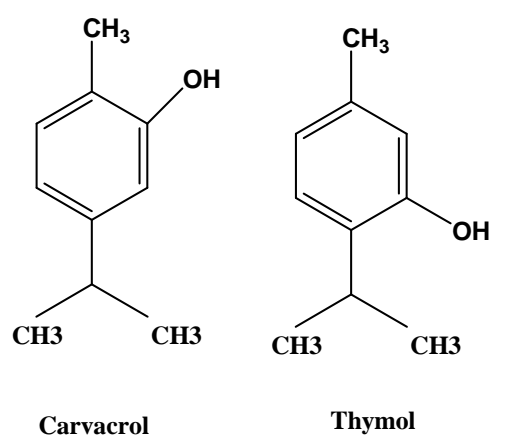

Figure 1 Chemical structures of phenolic compound (thymol and carvacrol)

effects (13). In Iranian traditional medicine, $Z M$ is used for carminative properties and analgesic. It is used for treatment of Candidiasis vaginitis and some pharmaceutical forms of this plant, such as syrups, drops, soft capsules and vaginal creams are sold as treatments for various diseases (2). Several studies analyzed carvacrol and thymol as the main compounds in the essential oil of ZM. Dehkordi et al. collected ZM from five different areas of Iran and analyzed its oils. According to the gas chromatography/mass spectrometry (GC-MS) data, the main oil constituents remained similar between plants from different geographical regions, but their relative quantities differed among plants from different regions. Thymol was the most abundant compound among all constituents in all samples (4).

In spite of the extensive uses of ZM in medicine and most of the pharmaceutical dosage forms, phytochemical investigations of ZM have been frequently focused on the essential oil and there are not any studies to standardized ZM hydroalcoholic extract to their thymol content and separation of thymol and carvacrol peaks by HPLC method.
However, type of solvent is affected on thymol extraction from extract. This study was performed for quantification of the thymol in different extracts of $Z M$ by HPLC method.

\section{Materials and Methods}

\section{Plant material}

The dried aerial parts of Zataria multiflora was collected from their major growing areas around Firozabad city of Fars province GO $\left(28.8194258^{\circ}\right.$ $\mathrm{N}, 52.5518705^{\circ} \mathrm{W}$ ), Iran at the full flowering stage (June and July 2012). It was confirmed by a senior botanist Prof. Mohammad Azadbakht at the Mazandaran University of Medical Science, Iran (Herbarium number: F-18-4-21). Five grams of the homogenous powder (1 $\mathrm{mm}$ in diameter mesh No. 18) with $50 \mathrm{ml}$ hydroalcoholic solvent with ethanol $34 \%, 42 \%$ and $70 \%$ for 48 hours. The solution was filtered through filter paper. Thymol and carvacrol standard materials were purchased from Merck (Germany) and Sigma (Germany) companies.

\section{Analysis}

The HPLC system consists of a model K-1001 solvent delivery system equipped with a Rheodyne injection valve (20 $\mu$ l sample loop inserted) and a $\mathrm{UV}-\mathrm{Vis}$ spectrophotometer detector model K-2600 set at $274 \mathrm{~nm}$ (all from Knauer Assoc., Germany). Analyze was performed by using an ODS-C18 column $(150 \times 4.6 \mathrm{~mm}$ i.d., $5 \mu \mathrm{m}$ particle size), and the corresponding guard column. All solvent were filtered and degassed earlier entering the column.

For separation of carvacrol (Merck, Germany) peak from thymol (Sigma, USA), different solvents as mobile 
phase in HPLC (methanol, acetic acid, water and acetonitrile) were used. Finally the mobile phase was selected an isocratic combination of acetonitrile: $\mathrm{H}_{2} \mathrm{O}$ : acetic acid (65:34:1). The mobile phase flow rate was $1.0 \mathrm{ml} / \mathrm{min}$, and all the measurements were done at ambient temperature. In this mobile phase, the peaks of thymol and carvacrol were successfully separated in the samples and pure peak of thymol was reached for quantification. Stock solutions of thymol $(0.5 \mathrm{mg} / \mathrm{ml})$ were prepared. Different concentrations $(0.025,0.05$, $0.1,0.25$ and $0.5 \mathrm{mg} / \mathrm{ml}$ ) were prepared from stock solution.

\section{Statistical analysis}

Data were presented as mean \pm standard deviation (SD) for three experiments. The results were analyzed by Excel software.

\section{Result}

The separation of thymol and carvacrol with reversed phase chromatography was provided with several mobile phases, because these phenols have similar chemical structures (Fig. 1). First we selected methanol-water mobile phase, however, thymol and carvacrol were not completely separated and with tailing in theirs chromatogram peaks. The best mobile phase was selected by varying proportion acetonitrile by adding acetic acid. Thymol and carvacrol was separated by best mobile phase of acetonitrile: $\mathrm{H}_{2} \mathrm{O}$ : acetic acid (65:34:1) at isocratic elution. In $\mathrm{ZM}$ extract, thymol and carvacrol were showed a retention times 10.4 and 9.8 minutes, respectively (Fig. 2). For finding of each peaks is on thymol or cravacrol, we injected thymol and carvacrol standards also addition of these standards to extract for adjustments of these phenols in Zatraia extract (Fig. 3).

Calibration graph was performed using the external standard technique following linear regression analysis by plotting concentration against peak area. Figure 3 shows the equations got for the calibration graphs and the regression coefficients $\left(r^{2}=0.998\right) \quad(\mathrm{Fig} 4)$. Samples of ZM extract (70, 42 and 34\% hydroalcoholic extract) were injected three times and chromatographs were reached. These samples were quantified from line equation of calibration curve, which was got from thymol standards. Table 1 showed the thymol in each hydroalcholic extracted samples. Thymol content was $2.7 \pm 0.06,3.7 \pm$ 0.07 and $6.0 \pm 0.11 \mathrm{mg} / \mathrm{g}$ dried extract for ethanol concentration of 34, 42 and $70 \%$, respectively.

\section{Discussion}

Z. multiflora has several biological and pharmacological properties. ZM is acted as an antimicrobial in the food industry (14-17) and used as an antispasmodic, anesthetic agent in Iranian traditional medicine (14). There are many studies that show that Z. multiflora has antibacterial (9) antifungal and antioxidant $(7,8)$ activities. Thymol acts on the microbial cell membrane and causes substantial morphological damage, resulting in a change in permeability and the release of cellular contents $(18,19)$. In previous studies, GC-MS instrument was performed for analysis the essential oil of ZM. The content of thymol in Zataria hydro alcoholic extract was analyzed by HPLC method, but the carvacrol peak was not identified in chromatography method (13). ZM is used as an important herbal medicine in Iranian traditional medicine for 1000 

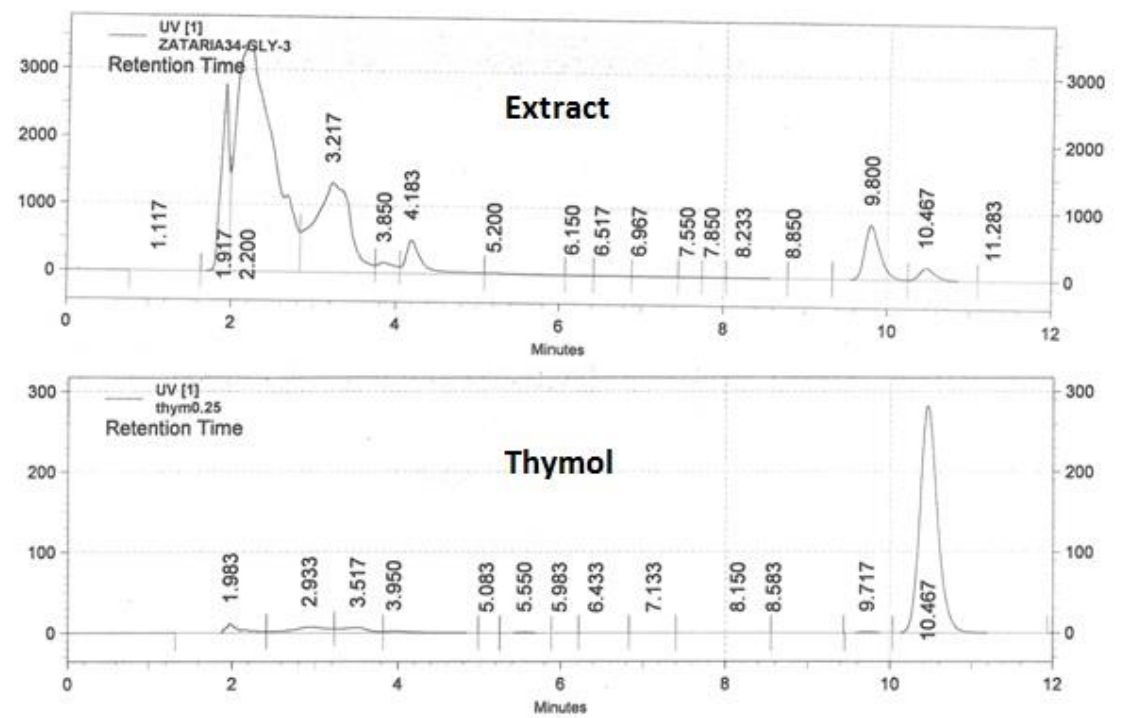

Figure 2 HPLC chromatograms of Zataria multiflora extract and thymol analyses with a mobile phase of acetonitrile: $\mathrm{H}_{2} \mathrm{O}$ : acetic acid (65:34:1).
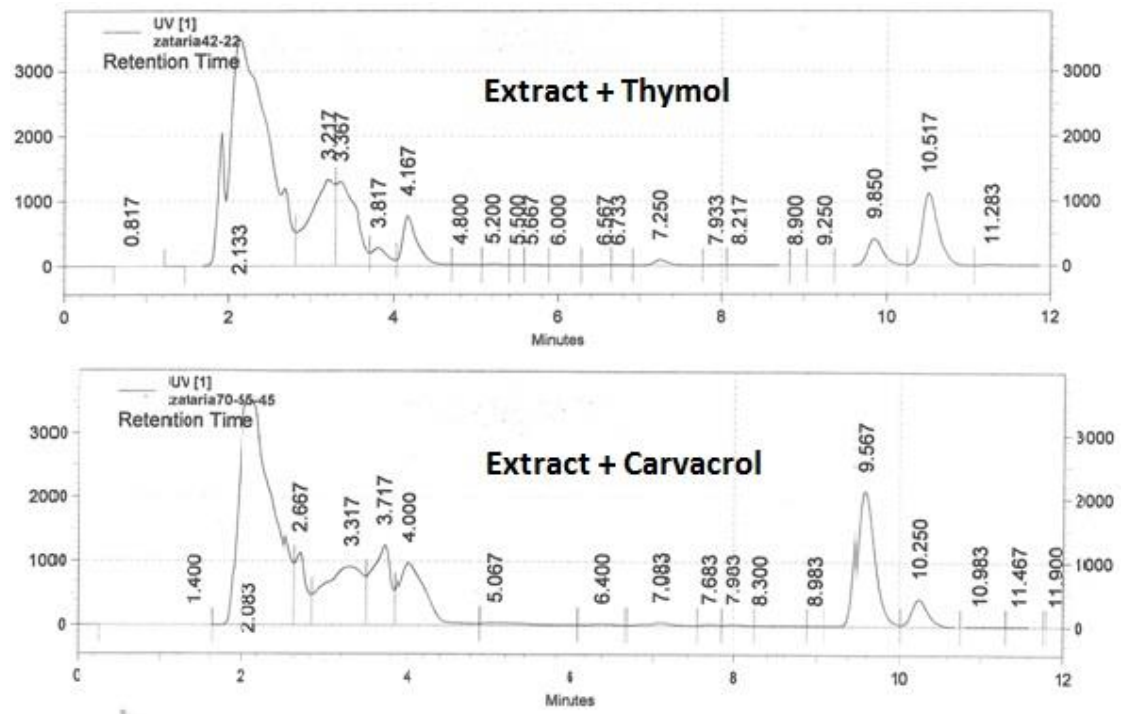

Figure 3 HPLC chromatograms of Zataria multiflora extract + thymol and Zataria multiflora extract + carvacrol that analyses with a mobile phase of acetonitrile: $\mathrm{H}_{2} \mathrm{O}$ : acetic acid (65:34:1) 


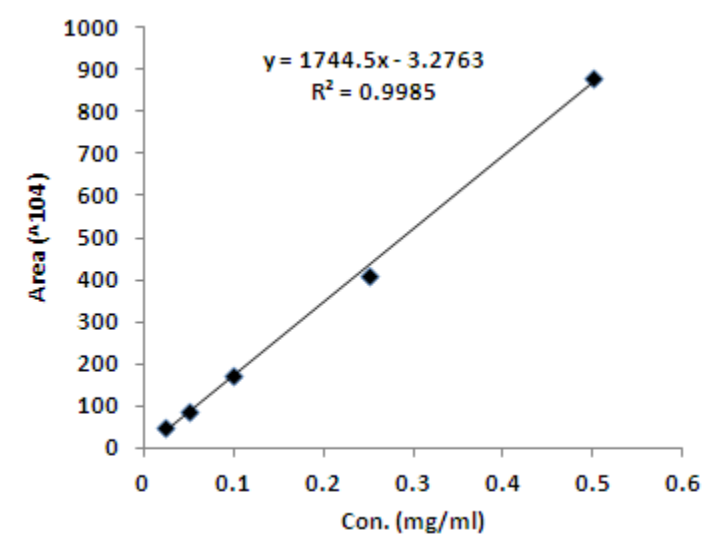

Figure 4 Thymol calibration curve

Table 1 Thymol content in different hyroalcoholic extract of Zataria multiflora extracted with different proportion of ethanol.

\begin{tabular}{lc}
\hline $\begin{array}{l}\text { Percentage of } \\
\text { ethanol for } \\
\text { extraction }\end{array}$ & $\begin{array}{c}\text { Thymol mg/g } \\
\text { extract } \\
\text { (mean } \pm \text { SD) }\end{array}$ \\
\hline 70 & $6.0 \pm 0.11$ \\
42 & $3.7 \pm 0.07$ \\
34 & $2.7 \pm 0.06$ \\
\hline
\end{tabular}

years to cure stomachache and agitation, and to combat insect bites (Canon of Medicine) (10) $\mathrm{ZM}$ can be natural therapeutic agents for the treatment of alzheimer's disease. Antioxidant, antiinflammatory, and anticholinesterase properties of thymol might contribute to its beneficial effects (20). Kavoosi showed that $Z M$ mitigated oxidative stress and may be used in the therapy of oxidative damage accompanying hyperglycemia and some inflammatory conditions (8). Thymol and carvacrol are isomeric non-polar phenolic compounds that only slightly soluble in water at neutral $\mathrm{pH}$, but it is extremely soluble in alcohols and other organic solvents. As results showed in figure 2, thymol has retention time about 1 minute more than carvacrol, it is showed thymol is more non-polar than carvacrol in chemical structure. Thymol and carvacrol have similar chemical structure with a difference in position of hydroxyl group (Fig. 1). Hydroxyl group is close to short chain of isopropyl for thymol, while this group is close to methyl group for carvacrol. The positions of hydroxyl group affect on polarity of these phenol. One of aims of our study was effects of percentage of ethanol on extraction of thymol from $\mathrm{ZM}$ herb, because thymol is most phenolic compound in this herb for pharmacological effects. Thymol (2isopropyl-5-methylphenol) is found in oil and extract of thyme and plants including Thymus vulgaris and $Z M$. Thymol is soluble in aqueous ethanol, and highly soluble in non-polar organic solvents, and the result of our study showed that the thymol content increase in Z. multiflora extract by rising of ethanol proportion. It was $2.7 \pm 0.06$ and $6.0 \pm 0.11 \mathrm{mg} / \mathrm{g}$ in ethanol 34 and $70 \%$, respectively.

\section{Conclusion}

In this study was established a mobile phase for separation of thymol and carvacrol as phenolic compounds in ZM by using HPLC method. This method is useful tool for assay thymol in ZM extract as pharmaceutical dosage form, which it is one of important herbal medicine. We showed that concentration of thymol in hydroalcoholic extract of $Z M$ is dependent to ethanol concentration in extraction solvent.

\section{Conflict of interest}

The authors declared no potential conflict of interest with respect to the authorship, and/or publication of this study. 


\section{References}

1. Hosseinzadeh H, Ramezani M, Salmani G. Antinociceptive, anti-inflammatory and acute toxicity effects of Zataria multiflora Boiss extracts in mice and rats. J Ethnopharmacol 2000;73:379-85.

2. Alizadeh NS, Khoei EM, Fazelimanesh M, Astaneh A. Antibacterial Effects of Zataria multiflora Boiss (Shirazi Avishan Extract) on Urinary Tract Escherichia coli Infections. Res J Biol Sci 2009;4:891-4.

3. Mahboubi M, Bidgoli FG. Antistaphylococcal activity of Zataria multiflora essential oil and its synergy with vancomycin. Phytomedicine 2010;17:548-50.

4. Saei-Dehkordi SS, Tajik H, Moradi M, KhalighiSigaroodi F. Chemical composition of essential oils in Zataria multiflora Boiss. from different parts of Iran and their radical scavenging and antimicrobial activity. Food Chem Toxicol 2010;48:1562-7.

5. Amanlou M, Beitollahi JM, Abdollahzadeh S, Tohidast-Ekrad Z. Miconazole gel compared with Zataria multiflora Boiss. gel in the treatment of denture stomatitis. Phytother Res 2006;20:966-9.

6. Ramezani M, Hosseinzadeh $\mathrm{H}$, Samizadeh S. Antinociceptive effects of Zataria multiflora Boiss fractions in mice. $\mathrm{J}$ Ethnopharmacol 2004;91:167-70.

7. Sharififar F, Moshafi M, Mansouri S, Khodashenas M, Khoshnoodi $\mathrm{M}$. In vitro evaluation of antibacterial and antioxidant activities of the essential oil and methanol extract of endemic Zataria multiflora Boiss. Food Control 2007; 18:800-5.

8. Kavoosi G, Teixeira da Silva JA, Saharkhiz MJ. Inhibitory effects of Zataria multiflora essential oil and its main components on nitric oxide and hydrogen peroxide production in lipopolysaccharide-stimulated macrophages. J Pharm Pharmacol 2012;64:1491-500.

9. Mahmoudabadi AZ, Dabbagh MA, Fouladi Z. In vitro anti-candida activity of Zataria multiflora Boiss. Evid Based Complement Alternat Med. 2007;4:351-3.

10. Sajed H, Sahebkar A, Iranshahi M. Zataria multiflora Boiss. (Shirazi thyme)--an ancient condiment with modern pharmaceutical uses. J Ethnopharmacol 2013;145:686-98.

11. Shokri H, Asadi F, Bahonar AR, Khosravi AR. The Role of Zataria multiflora essence (Iranian herb) on innate immunity of animal model. Iran J Immunol 2006;3:164-8.

12. Jaffary F, Ghannadi A, Siahpoush A. Antinociceptive effects of hydroalcoholic extract and essential oil of Zataria multiflora. Fitoterapia 2004;75:217-20.
13. Hosseinimehr SJ, Mahmoudzadeh A, Ahmadi A, Ashrafi SA, Shafaghati N, Hedayati N. The radioprotective effect of Zataria multiflora against genotoxicity induced by gamma irradiation in human blood lymphocytes. Cancer Biother Radiopharm 2011;26:325-9.

14. Fazeli MR, Amin G, Attari M, Ashtiani H, Jamalifar H, Samadi N. Antimicrobial activities of Iranian sumac and avishan-e shirazi (Zataria multiflora) against some food-borne bacteria. Food Control 2007;18:646-9.

15. Abbasifar A, Akhoundzade BA, Karim G, Misaghi A, Bokaei S, Gandomi NA, et al. Evaluation of Zataria mutiflora Boiss. Effect on Staphylococcus aureus in feta cheese. J Med Plants 2008:7:105-15.

16. Gandomi H, Misaghi A, Basti AA, Bokaei S, Khosravi A, Abbasifar A, et al. Effect of Zataria multiflora Boiss. essential oil on growth and aflatoxin formation by Aspergillus flavus in culture media and cheese. Food Chem Toxicol 2009;47:2397-400.

17. Ghasemi S, Javadi NS, Moradi M, Oromiehie A, Khosravi-Darani K. Investigation on Development of zein antimicrobial edible film and essential oil of Zataria multiflora Boiss. on Sallmonella enteritidis, listeria monocytogenes, escherichia coli and staphylococcus aureus. Asian J Chem 2012;24:5941-2.

18. Moosavy MH, Akhondzadeh Basti A, Misaghi A, Salehi TZ, Abbasifar R, Mousavi H, et al. Effect of Zataria multiflora Boiss. essential oil and nisin on Salmonella typhimurium and Staphylococcus aureus in a food model system and on the bacterial cell membranes. Food Res Internat 2008;41:1050-7.

19. Di Pasqua R, Hoskins N, Betts G, Mauriello G. Changes in membrane fatty acids composition of microbial cells induced by addiction of thymol, carvacrol, limonene, cinnamaldehyde, and eugenol in the growing media. J Agric Food Chem 2006;54:2745-9.

20. Majlessi N, Choopani S, Kamalinejad M, Azizi Z. Thymol as a main constituent of Zataria Multiflora boiss: essential oil attenuates amyloid $\beta$-induced cognitive deficits in a rat model of Alzheimer's disease. Alzheimers Dementia 2011;7:770. 